\title{
ON MULTIPLE POSITIVE SOLUTIONS OF POSITONE AND NON-POSITONE PROBLEMS
}

\author{
F. J. S. A. CORRÊA
}

Received 7 January 1999

\section{Introduction}

In this paper, we consider the following problem:

$$
-\triangle u=f(u) \quad \text { in } \Omega, u=0 \text { on } \partial \Omega,
$$

where $\Omega$ is the ball $B_{R}=\left\{x \in \mathbb{R}^{N} ;|x|<R\right\},|\cdot|$ is the Euclidean norm in $\mathbb{R}^{N}$, and $f: \mathbb{R}^{+} \rightarrow \mathbb{R}$ is a locally Lipschitzian continuous function. We are concerned with two classes of problems, namely,

(i) the positone problem: $f(0) \geq 0$;

(ii) the non-positone problem: $f(0)<0$.

The study of positone problems was initiated by Keller and Cohen [14], see also [15], motivated by problems arose from the theory of nonlinear heat generation. In the past twenty five years there has been considerable interest in this class of semilinear elliptic boundary value problems and there is a wide literature on this subject. The reader may consult the survey by Lions [17], and the references therein, where many interesting questions are studied under a different point of view.

In the positone case we consider the following assumptions:

(f1) there exist $0<a_{1}<a_{2}<a_{3}$ so that $f\left(a_{1}\right)=f\left(a_{2}\right)=0$ and $F\left(a_{3}\right)>F\left(a_{1}\right)$, where $F(t)=\int_{0}^{t} f$

(f2) $|f(t)| \leq \alpha$, for all $t \in \mathbb{R}^{+}$, for some constant $\alpha>0$;

(P) $f(0)>0$ or, if $f(0)=0$, then $f_{+}^{\prime}(0)>0$, where $f_{+}^{\prime}(0)=\lim _{t \rightarrow 0^{+}}(f(t) / t)$ and prove the result below.

Theorem 1.1. Suppose that $f: \mathbb{R}^{+} \rightarrow \mathbb{R}$ satisfies ( $\left.f 1\right)$, ( $\left.f 2\right)$, and $(P)$. If $R$ is sufficiently large, problem (1.1) possesses at least three radial positive solutions $u_{1}, u_{2}$, and $u_{3}$ such that $\partial u_{i} / \partial r<0$, for $i=1,2,3$ and $0<r<R$. In particular

$$
0<\left|u_{1}\right|_{\infty}<a_{1}<a_{2}<\left|u_{2}\right|_{\infty}
$$

where $|\cdot|_{\infty}$ is the sup norm.

Copyright $\odot 1999$ Hindawi Publishing Corporation Abstract and Applied Analysis 4:2 (1999) 101-108 1991 Mathematics Subject Classification: 35J65, 34B15 URL: http://aaa.hindawi.com/volume-4/S1085337599000044.html 
Remark 1.2. Some results like the one above have been obtained by several authors when the area condition $F\left(a_{3}\right)>F\left(a_{1}\right)$ has been considered. This condition was first used by Brown and Budin [3] who proved a result similar to Theorem 1.1 by using a combination of variational and monotone iteration methods. Later Hess [13] studied this problem by using variational methods and degree theory. In [9], the above condition is also used and, by using solely variational methods, the existence of three ordered positive solutions is shown. These authors studied the case in which $f$ has a third root, that is, $f\left(a_{3}\right)=0$. In Theorem 1.1 this assumption is not made and we use only variational methods. Also, Theorem 1.1 is used as an essential tool in the study of non-positone case.

The non-positone case has been studied in recent years mainly by Brown, Castro, Shivaji, Arcoya, and Calahorrano, among others. See, for example, [2, 4, 5]. In this case the following assumption is posed:

$$
f(0)<0 .
$$

Motivated by the study of discontinuous nonlinear problems we consider, as in [2], the following multivalued problem:

$$
-\triangle u(x) \in \hat{f}(u(x)) \text { a.e in } \Omega, u=0 \text { on } \partial \Omega,
$$

where $\hat{f}$ is the multivalued function defined by

$$
\hat{f}(t)= \begin{cases}0, & \text { if } t<0 \\ {[f(0), 0],} & \text { if } t=0 \\ f(t), & \text { if } t>0\end{cases}
$$

By a solution of (1.4), we mean a function $u \in C^{1}(\bar{\Omega}) \cap C^{2}\left(\Omega^{*}(u)\right)$ with $\Omega^{*}(u)=$ $\{x \in \Omega ; u(x) \neq 0\}$ and verifying (1.4).

Remark 1.3. Some authors (cf. Chang [6]) have treated discontinuous problems by using a direct variational approach. In the present case, we consider the multivalued problem (1.4) as the limit of smooth approximating problems in order to use Theorem 1.1. Thus two nonnegative solutions $u_{0}$ and $v_{0}$, in the sense of (1.4), are obtained as limits of smooth approximating solutions. Then we use the symmetry results in [11] and the maximum principle to show that $u_{0}$ and $v_{0}$ are positive classical solutions of (1.1). For this a crucial step is to show that the set $\Omega_{0}(u)=\Omega / \Omega^{*}(u)$ has null Lebesgue measure for $u=u_{0}, v_{0}$.

The following assumptions on $f$ are considered:

(f3) there exists $\theta>0$ such that $f(t)<0$ if $0 \leq t<\theta$ and $f(\theta)=0$,

(f4) there is $a>\theta$ satisfying $F(a)>0$.

We are now ready to state the following theorem.

Theorem 1.4. Suppose that $f: \mathbb{R}^{+} \rightarrow \mathbb{R}$ is a bounded $C^{1}$-function satisfying (f3), (f4), and (1.3). Then problem (1.4) has at least two radial positive solutions $u_{0}$ and $v_{0}$ if $R$ 
is sufficiently large. In fact $\Omega_{0}\left(u_{0}\right)=\Omega_{0}\left(v_{0}\right)=\phi$, which implies $u_{0}$ and $v_{0}$ are radial classical positive solutions of (1.1) such that $\partial u / \partial r<0$, for $0<r<R$ and $u=v_{0}, u_{0}$.

Remark 1.5. It is well known in the positone case, see, for example, Cohen and Laetsch [7], that if $f$ is concave, problem (1.1) has at most one positive solution, but may have multiple solutions when $f$ is convex. In contrast with this case Theorem 1.4 shows that in the non-positone problem we may obtain multiplicity of positive solutions with $f$ concave. The function $f(t)=\alpha-e^{-t}, 0<\alpha<1$ and $t \geq 0$, is a simple example of a bounded concave function satisfying (f3), (f4), and (1.3).

\section{Proof of Theorem 1.1}

First solution. Let us consider the following function:

$$
f_{1}(t)= \begin{cases}f(0), & \text { if } t \leq 0 \\ f(t), & \text { if } 0 \leq t \leq a_{1} \\ 0, & \text { if } a_{1} \leq t\end{cases}
$$

and the functional $I_{1}: E \rightarrow \mathbb{R}, E:=H_{0}^{1}(\Omega)$ with the usual norm $\|u\|^{2}=\int|\nabla u|^{2}$, where the integrals are taken over all $\Omega$, unless we state the contrary, defined by

$$
I_{1}(u)=\frac{1}{2} \int|\nabla u|^{2}-\int F_{1}(u), \quad F_{1}(u)=\int_{0}^{u} f_{1} .
$$

Note that $I_{1}$ is the Euler-Lagrange functional associated to the problem

$$
-\triangle u=f_{1}(u) \quad \text { in } \Omega, u=0 \text { on } \partial \Omega .
$$

Since $I_{1}$ is coercive and weakly lower semi-continuous (w.l.s.c.), see [10], it achieves its minimum at some point $u_{1} \in E$, which is a weak solution of (2.3) and a bootstrap argument shows that $u_{1}$ is a classical solution of (2.3). Assumption (P) implies that $\liminf _{t \rightarrow 0^{+}}(f(t) / t)>\lambda_{1}:=\lambda_{1}(R)$, if $R$ is sufficiently large, where $\lambda_{1}$ is the first eigenvalue of $\left(-\triangle, H_{0}^{1}(\Omega)\right)$. In particular, this yields $I_{1}\left(u_{1}\right)<0$, that is, $u_{1} \neq \equiv 0$ in $\Omega$. So the maximum principle provides $0<u_{1}(x)<a_{1}$ in $\Omega$ and then $u_{1}$ satisfies (1.1). Moreover, it is easy to show that

$$
I_{1}\left(u_{1}\right)>-K_{N} F\left(a_{1}\right) R^{N},
$$

where $K_{N}$ is a positive constant depending only on $N$. Actually (2.4) is valid for all $u \in E$ satisfying $0<u(x)<a_{1}$ a.e. in $\Omega$.

Second solution. We now consider the function $I: E \rightarrow \mathbb{R}$ given by

$$
I(u)=\frac{1}{2} \int|\nabla u|^{2}-\int F(u), \quad F(u)=\int_{0}^{u} f,
$$

where we still denote by $f$ the extension of the former function $f$ and defined by

$$
f(t)= \begin{cases}f(0), & \text { if } t \leq 0 \\ f(t), & \text { if } 0<t\end{cases}
$$


104 On multiple positive solutions of positone and non-positone problems

A standard calculation shows that $I$ is coercive and w.l.s.c. Hence $I$ attains its minimum at $u_{2} \in E$. We may not guarantee, up to now, that $u_{1} \neq u_{2}$. For this we consider the following function used by Klaasen and Mitidieri [16]:

$$
u_{R}(x)= \begin{cases}a_{3}, & \text { if }|x| \leq R-1 \\ (R-|x|) a_{3}, & \text { if } R-1<|x|<R\end{cases}
$$

Setting $C_{R}=\left\{x \in \mathbb{R}^{N} ; R-1<|x|<R\right\}$ we obtain

$$
\begin{aligned}
I\left(u_{R}\right) & \leq \frac{1}{2} \int_{C_{R}}\left|\nabla u_{R}\right|^{2}-\int_{B_{R-1}} F\left(a_{3}\right)-\int_{C_{R}} F\left(u_{R}\right) \\
& \leq \frac{a_{3}^{2}}{2}\left|C_{R}\right|-F\left(a_{3}\right)\left|B_{R-1}\right|+C_{1}\left|C_{R}\right| \\
& \leq \frac{a_{3}^{2}}{2} h_{N} R^{N-1}-F\left(a_{3}\right) K_{N}(R-1)^{N}+C_{1} h_{N} R^{N-1} .
\end{aligned}
$$

Thus

$$
I\left(u_{R}\right) \leq C_{N} R^{N-1}-K_{N} F\left(a_{3}\right) R^{N},
$$

where the constants $C_{1}, K_{N}, h_{N}$, and $C_{N}$ do not depend on $R$. Since $F\left(a_{3}\right)-F\left(a_{1}\right)>0$ one has, for $R$ sufficiently large,

$$
K_{N}\left(F\left(a_{3}\right)-F\left(a_{1}\right)\right) R^{N}>C_{N} R^{N-1}
$$

which implies

$$
I\left(u_{R}\right)<-K_{N} F\left(a_{1}\right) R^{N}<I_{1}\left(u_{1}\right)=I\left(u_{1}\right) .
$$

Then the minimum $u_{2}$ of $I$ satisfies

$$
I\left(u_{2}\right) \leq I\left(u_{R}\right)<I\left(u_{1}\right)
$$

This shows that $u_{1} \neq u_{2}$. A bootstrap argument guarantees that $u_{2} \in C^{2, \alpha}(\bar{\Omega})$ and the maximum principle implies that $0<u_{2}(x)$, for all $x \in \Omega$, and $a_{2}<\left|u_{2}\right|_{\infty}$, because $I\left(u_{2}\right)<-K_{N} F\left(a_{1}\right) R^{N}<I(u)$, for all $u$ so that $0<u(x)<a_{1}$ in $\Omega$.

Third solution. Using an argument as in de Figueiredo [10] we may prove that $u_{1}$ is a local minimum of $I$. A straightforward computation shows that $I$ satisfies the Palais-Smale condition, see [1]. So by the Ambrosetti and Rabinowitz's Mountain Pass Theorem, see [1], we find a third solution (positive) $u_{3}$ of (1.1) satisfying

$$
I\left(u_{3}\right) \geq-K_{N} F\left(a_{1}\right) R^{N}
$$


Finally we observe, in view of symmetry results in Gidas, Ni, and Nirenberg [11], that $u_{i}, i=1,2,3$, are radial and $\partial u_{1} / \partial r<0, i=1,2,3$, for all $0<r<R$. This proves Theorem 1.1.

Remark 2.1. If $f$ is decreasing on $\left[0, a_{1}\right]$, there exists only the solution $u_{1}$ such that $0<u_{1}(x)<a_{1}$. Hence, in this case, $u_{3}$ also satisfies $a_{2}<\left|u_{3}\right|_{\infty}$. In fact the Cosner and Schmitt's result, see [8], implies that $\left|u_{2}\right|_{\infty},\left|u_{3}\right|_{\infty}>a_{3}$.

\section{Proof of Theorem 1.4}

To prove Theorem 1.4 we use mainly Theorem 1.1.

For this, we first consider smooth approximations of $\hat{f}$ given by

$$
f_{n}(t)= \begin{cases}f(t), & \text { if } \frac{1}{n} \leq t \\ f_{n}(t) \geq f(t), & \text { if } 0 \leq t \leq \frac{1}{n} \\ f_{n}(t)=\frac{1}{n}, & \text { if } t \leq 0\end{cases}
$$

$f_{n}$ is $C^{1}$, there is $a_{1 n} \in(0,1 / n)$ such that $F_{n}(a)>F_{n}\left(a_{1 n}\right)$ and $f_{n}(t) \rightarrow f(t)$, for all $t>0$. As usual $F_{n}(t)=\int_{0}^{t} f_{n}$. Let us now consider the problem

$$
-\triangle u=f_{n}(u) \quad \text { in } \Omega, u=0 \text { on } \partial \Omega .
$$

In order to apply Theorem 1.1 to problem (3.2) we consider the functional $I_{n}$ on $E$ defined by

$$
I_{n}(u)=\frac{1}{2} \int|\nabla u|^{2}-\int F_{n}(u)
$$

Since $f_{n}: \mathbb{R} \rightarrow \mathbb{R}$ satisfies the assumptions of Theorem 1.1, problem (3.2) possesses at least three positive solutions $u_{1 n}, u_{2 n}$, and $u_{3 n}$. In particular

$$
I_{n}\left(u_{2 n}\right) \leq C_{N} R^{N-1}-K_{N} F_{n}(a) R^{N}<-K_{N} F_{n}\left(a_{1 n}\right) R^{N} \leq I_{n}\left(u_{3 n}\right) .
$$

Elliptic regularity yields $\left|u_{2 n}\right|_{w^{2, p},},\left|u_{3 n}\right|_{w^{2, p}} \leq C_{p}$, for all $p \geq 1$. For $p>N$ one has $u_{2 n} \rightarrow u_{0}$ and $u_{3 n} \rightarrow v_{0}$ in $\mathrm{C}^{1, \alpha}(\bar{\Omega})$ for some $0<\alpha<1$, eventually for subsequences. Since $F_{n}(a) \rightarrow F(a)$ and $F_{n}\left(a_{1 n}\right) \rightarrow 0$ as $n \rightarrow \infty$, one has

$$
\begin{aligned}
\int\left|\nabla u_{2 n}\right|^{2} & \longrightarrow \int\left|\nabla u_{0}\right|^{2}, & & \int\left|\nabla u_{3 n}\right|^{2} \rightarrow \int\left|\nabla v_{0}\right|^{2}, \\
\int F\left(u_{2 n}\right) & \longrightarrow \int F\left(u_{0}\right), & & \int F\left(u_{3 n}\right) \longrightarrow \int F\left(v_{0}\right) .
\end{aligned}
$$

Taking limits in the inequalities in (3.4) we obtain

$$
I\left(u_{0}\right) \leq C_{N} R^{N-1}-K_{N} F(a) R^{N} \leq I\left(v_{0}\right) .
$$


106 On multiple positive solutions of positone and non-positone problems

Noticing that $-K_{N} F_{n}\left(a_{1 n}\right) R^{N} \leq I_{n}\left(u_{3 n}\right)$ and $F_{n}\left(a_{1 n}\right) \rightarrow 0$ one has $0 \leq I\left(v_{0}\right)$. Because $F(a)>0$ we obtain $C_{N} R^{N-1}-K_{N} F(a) R^{N}<0$ if $R$ is sufficiently large.

Thus

$$
I\left(u_{0}\right) \leq C_{N} R^{N-1}-K_{N} F(a) R^{N}<0 \leq I\left(v_{0}\right)
$$

and so $u_{0} \neq v_{0}$.

We show that $u_{0}$ and $v_{0}$ are nontrivial nonnegative solutions of (1.4). First we observe that $f_{n}$ may be chosen decreasing in $(0,1 / n)$, for each $n=1,2, \ldots$. Thus problem (3.2) possesses a unique positive solution $u_{1 n}$ satisfying $0<u_{1 n}(x)<1 / n$, for each $n=1,2, \ldots$. Hence $\left|u_{2 n}\right|_{\infty},\left|u_{3 n}\right|_{\infty}>\theta$, for all $n=1,2, \ldots$ In fact, the result in Cosner-Schmitt [8] implies that $\left|u_{2 n}\right|_{\infty},\left|u_{3 n}\right|_{\infty} \geq a$ for all $n=1,2, \ldots$. Consequently, $\left|u_{0}\right|_{\infty},\left|v_{0}\right|_{\infty} \geq a$ and $u_{0}$ and $v_{0}$ are not identically zero.

If $t_{n}>0$ and $t_{n} \rightarrow t>0$, then $f_{n}\left(t_{n}\right) \rightarrow f(t)$. From now on, we set $u=u_{0}$ or $u=v_{0}$. Thus if $x \in \Omega^{*}(u)$ one has $\lim _{n \rightarrow \infty} f_{n}\left(u_{n}(x)\right)=f(u(x))$, where $u_{n}=u_{2 n}$ or $u_{n}=u_{3 n}$. Taking $\varphi \in C_{0}^{\infty}\left(\Omega^{*}(u)\right)$ and using the fact that

$$
-\triangle u_{n}(x)=f_{n}\left(u_{n}(x)\right) \text { in } \Omega, u_{n}=0 \text { on } \partial \Omega,
$$

we obtain

$$
\int_{\Omega^{*}} \nabla u_{n} \cdot \nabla \varphi=\int_{\Omega^{*}(u)} f_{n}\left(u_{n}\right) \varphi \Longrightarrow \int_{\Omega^{*}(u)} \nabla u \cdot \nabla \varphi=\int_{\Omega^{*}(u)} f(u) \varphi .
$$

Consequently, $u \in C^{2}\left(\Omega^{*}(u)\right)$ and $-\triangle u=f(u)$ in $\Omega^{*}(u)$. In $\Omega_{0}(u)$ we have, as a consequence of a well-known result by Stampacchia (cf. [15, Lemma A.4]), $-\Delta u(x)=$ $0 \in[f(0), 0]$ a.e., and so $u_{0}$ and $v_{0}$ are both nontrivial nonnegative solutions of (1.4). Let us now prove that if $u\left(x_{0}\right)=0$ for some $\left|x_{0}\right|=r<R$, it follows that $u(x)=0$ for all $r<|x|<R$. Actually, if $u(x)>0$ for some $r<|x|<R$, then $(\partial u / \partial r)(y)>0$, for some $r<|y|<R$, which is a contradiction in view of $(\partial u / \partial r)(x) \leq 0$ if $0<|x|<R$. Remember that $\partial u_{n} / \partial r<0$ for $0<r<R$ which implies that $\partial u / \partial r \leq 0$ because $u_{n} \rightarrow u$ in $C^{1, \alpha}(\bar{\Omega})$. So $\Omega_{0}(u)$ is a set like $A_{\rho}=B_{R}-B_{R-\rho}$, for some $\rho \geq 0$. Suppose that $\rho>0$. In view of $f(u) \in C^{1}\left(\bar{B}_{R-\rho}\right)$ one has

$$
-\triangle\left(\frac{\partial u}{\partial r}\right)=f^{\prime}(u) \frac{\partial u}{\partial r} \quad \text { in } B_{R-\rho} .
$$

Since $f$ is $C^{1}$ there is $\mu>0$ satisfying $f^{\prime}(t)+\mu \geq 0$, for all $t \in[0, M]$, with $M=$ $\max \left\{\left|u_{0}\right|_{\infty},\left|v_{0}\right|_{\infty}\right\}$. Consequently,

$$
-\triangle\left(-\frac{\partial u}{\partial r}\right)+\mu\left(-\frac{\partial u}{\partial r}\right)=\left(f^{\prime}(u)+\mu\right)\left(-\frac{\partial u}{\partial r}\right) \text { in } B_{R-\rho} .
$$

Therefore, in view of $\partial u / \partial r=0$ in $A_{\rho}$ we have by applying the generalized Green's formula on $B_{R-\rho}$ that

$$
-\triangle\left(-\frac{\partial u}{\partial r}\right)+\mu\left(-\frac{\partial u}{\partial r}\right) \geq 0 \quad \text { in } \Omega
$$

in the weak sense and as $u>0$ in $B_{R-\rho}$ we have $\partial u / \partial r \not \equiv 0$ and so, by using the generalized maximum principle, (cf. [12, Theorem 8.19]), $\partial u / \partial r<0$ in $\Omega$, which is a 
contradiction with assumption $\rho>0$. Hence, $\rho=0$. This implies that $\Omega_{0}(u)=\phi$, then $u>0$ in $\Omega$ and $\partial u / \partial r<0$ if $0<r<R$. It follows that $u_{0}$ and $v_{0}$ are classical solutions of (1.1). This concludes the proof of Theorem 1.4.

\section{Acknowledgement}

The author was partially supported by CNPq/Brazil.

\section{References}

[1] A. Ambrosetti and P. H. Rabinowitz, Dual variational methods in critical point theory and applications, J. Functional Analysis 14 (1973), 349-381. MR 51\#6412. Zbl 273.49063.

[2] D. Arcoya and M. Calahorrano, Multivalued non-positone problems, Atti Accad. Naz. Lincei Cl. Sci. Fis. Mat. Natur. Rend. (9) Mat. Appl. 1 (1990), no. 2, 117-123. MR 91j:35101. Zbl 719.35024.

[3] K. J. Brown and H. Budin, On the existence of positive solutions for a class of semilinear elliptic boundary value problems, SIAM J. Math. Anal. 10 (1979), no. 5, 875-883. MR 82k:35043. Zbl 414.35029.

[4] K. J. Brown, A. Castro, and R. Shivaji, Nonexistence of radially symmetric nonnegative solutions for a class of semi-positone problems, Differential Integral Equations 2 (1989), no. 4, 541-545. MR 90f:35010. Zbl 736.35039.

[5] K. J. Brown and R. Shivaji, Instability of nonnegative solutions for a class of semipositone problems, Proc. Amer. Math. Soc. 112 (1991), no. 1, 121-124. MR 91h:35047. Zbl 741.35003.

[6] K. C. Chang, Variational methods for nondifferentiable functionals and their applications to partial differential equations, J. Math. Anal. Appl. 80 (1981), no. 1, 102-129. MR 82h:35025. Zbl 487.49027.

[7] D. S. Cohen and T. W. Laetsch, Nonlinear boundary value problems suggested by chemical reactor theory, J. Differential Equations 7 (1970), 217-226. MR 41\#3994. Zbl 201.43102.

[8] C. Cosner and K. Schmitt, A priori bounds for positive solutions of a semilinear elliptic equation, Proc. Amer. Math. Soc. 95 (1985), no. 1, 47-50. MR 86h:35045. Zbl 577.35041.

[9] D. G. de Figueiredo, On the existence of multiple ordered solutions of nonlinear eigenvalue problems, Nonlinear Anal. 11 (1987), no. 4, 481-492. MR 88k:35157. Zbl 661.35070.

[10] _ Lectures on the Ekeland Variational Principle with Applications and Detours, Tata Institute of Fundamental Research Lectures on Mathematics and Physics, vol. 81, Published for the Tata Institute of Fundamental Research, Bombay; by Springer-Verlag, Berlin, New York, 1989, Lectures delivered at the Indian Institute of Science, Bangalore, India under the T.I.F.R.-I.I.Sc. programme in applications of mathematics. MR 90j:49006. Zbl 688.49011.

[11] B. Gidas, W. M. Ni, and L. Nirenberg, Symmetry and related properties via the maximum principle, Comm. Math. Phys. 68 (1979), no. 3, 209-243. MR 80h:35043. Zbl 425.35020.

[12] D. Gilbarg and N. S. Trudinger, Elliptic Partial Differential Equations of Second Order, Grundlehren der mathematischen Wissenschaften, vol. 224, Springer-Verlag, Berlin, Heidelberg, New York, 1977. MR 57\#13109. Zbl 361.35003.

[13] P. Hess, On multiple positive solutions of nonlinear elliptic eigenvalue problems, Comm. Partial Differential Equations 6 (1981), no. 8, 951-961. MR 82j:35062. Zbl 468.35073.

[14] H. B. Keller and D. S. Cohen, Some positone problems suggested by nonlinear heat generation, Bifurcation Theory and Nonlinear Eigenvalue Problems (New York, Amsterdam) (J. B. Keller and S. Antman, eds.), W. A. Benjamin, Inc., 1969. 
108 On multiple positive solutions of positone and non-positone problems

[15] D. Kinderlehrer and G. Stampacchia, An Introduction to Variational Inequalities and their Applications, Pure and Applied Mathematics, vol. 88, Academic Press Inc. [Harcourt Brace Jovanovich Publishers], New York, London, 1980. MR 81g:49013. Zbl 457.35001.

[16] G. A. Klaasen and E. Mitidieri, Standing wave solutions for a system derived from the FitzHugh-Nagumo equations for nerve conduction, SIAM J. Math. Anal. 17 (1986), no. 1, 74-83. MR 87b:35087. Zbl 593.35043.

[17] P.-L. Lions, On the existence of positive solutions of semilinear elliptic equations, SIAM Rev. 24 (1982), no. 4, 441-467. MR 84a:35093. Zbl 511.35033.

F. J. S. A. Corrêa: Departamento de Matemática-C.C.E.N, Universidade Federal do PARÁ, 66.075-900-BelÉM-PA, BRAZIL

E-mail address: fjulio@ufpa.br 


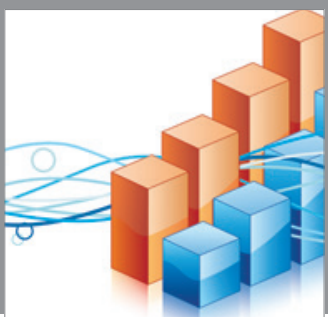

Advances in

Operations Research

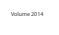

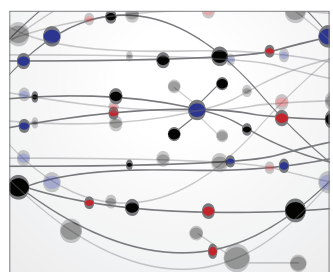

\section{The Scientific} World Journal
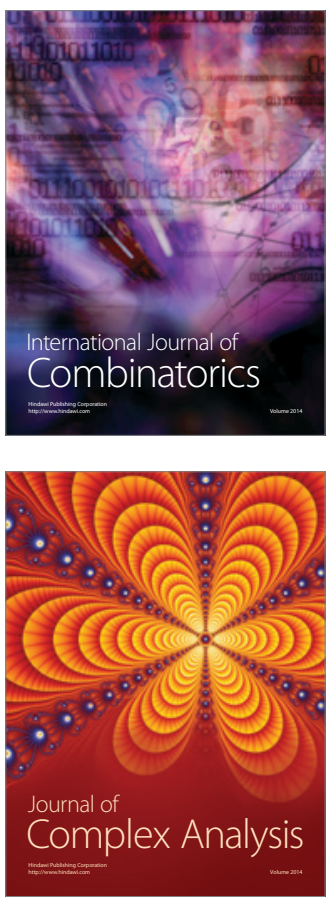

International Journal of

Mathematics and

Mathematical

Sciences
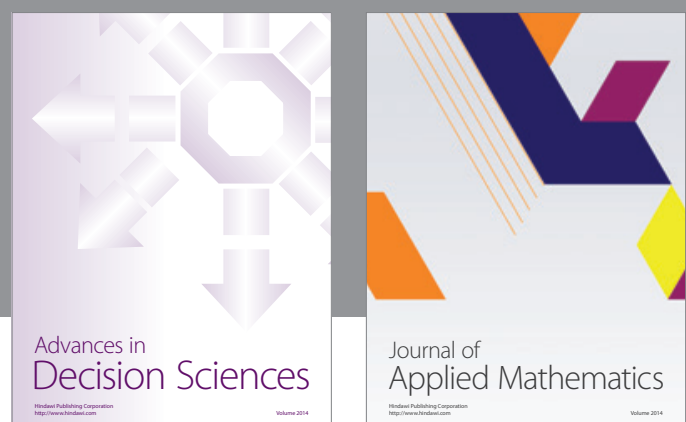

Journal of

Applied Mathematics
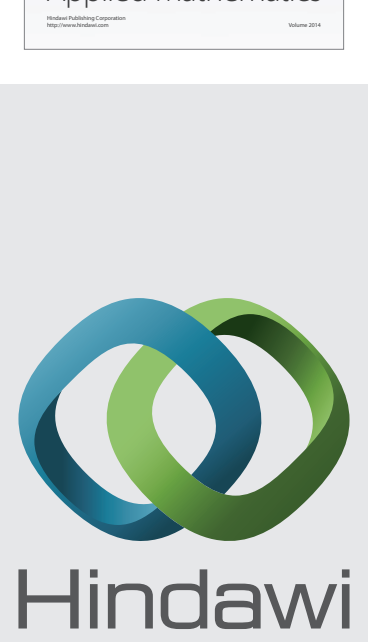

Submit your manuscripts at http://www.hindawi.com
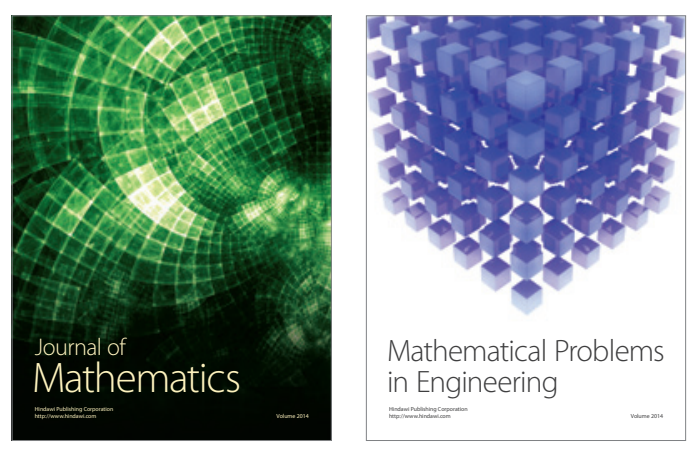

Mathematical Problems in Engineering
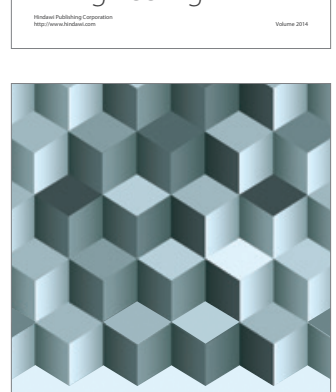

Journal of

Function Spaces
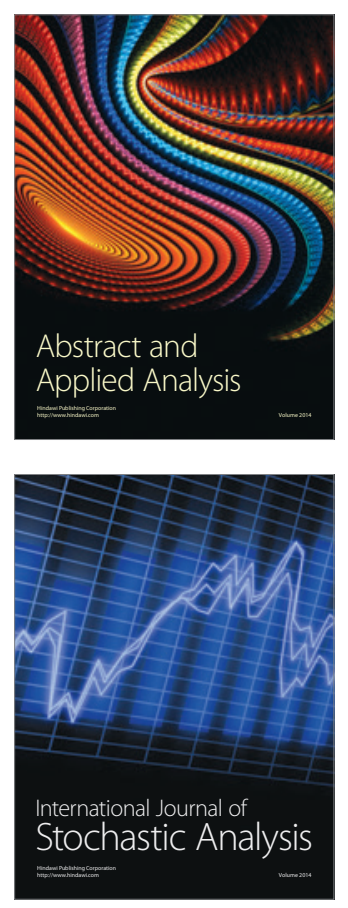

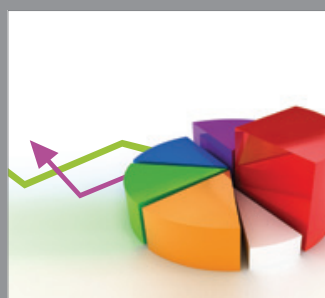

ournal of

Probability and Statistics

Promensencen
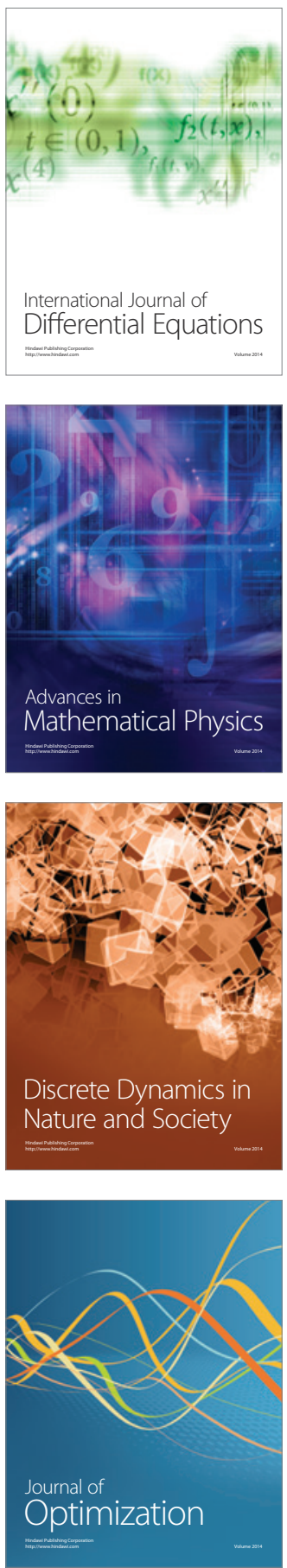